\title{
COMENTARIO ESTILÍSTICO DE UN SONETO DE QUEVEDO
}

José Domínguez Caparrós

U.N.E.D.

Comienzo precisando que el comentario que voy a hacer es un comentario estilístico. Por tanto, será un comentario literario en tanto en cuanto convengamos que el texto que voy a examinar es un texto literario. Pues lo literario es un concepto que no deja de plantear problemas: no todo el mundo en todas las épocas considera literarios los mismos textos. Por otra parte, un texto que se considera literario puede ser analizado desde distintos puntos de vista: histórico, histórico-literario, filosófico, psicológico, lingüistico... Con esto quiero decir que lo literario debe ir siempre precisado por un adjetivo: histórico, estilístico u otro.

Pues bien, yo voy a hacer un comentario estilístico-literario. Comentario estilístico es aquel que toma el lenguaje del texto como punto de partida para analizar dicho texto. Por eso es normal analizar el texto guiándose por los mismos niveles de análisis que la lingüística diferencia en todo texto: el fónico, el morfosintáctico, el semántico y el referencial. No quiero repetir lo ya expuesto en mi trabajo Introducción al comentario de textos, página 32 , ni la fundamentación de este esquema en el trabajo de Emile Benveniste "Los niveles del análisis lingüístico».

Otra aclaración referida a la exposición del comentario. Quiero señalar que en ningún caso debe pensarse que lo que yo digo responde exactamente a lo que ocurre realmente en la mente de todo lector del poema. El comentario no es una lectura ingenua, sino trabajada con determinados instrumentos. Quizá algunas de las cosas que diga son percibidas por todo lector en una primera lectura; otras, sin embargo, lo podrán ser sólo por algunos lectores; mientras que habrá quizá algunas que pertenezcan sola y exclusivamente a mi percepción particular del texto.

Tengo, pues, dos posibilidades: exponer mis apreciaciones según el orden del análisis (fonética, sintaxis, semántica y designación); o exponer mis apreciaciones según un supuesto orden de lectura (comentario de todos los aspectos del primer verso, luego del segundo y así sucesivamente). Pero esta segunda manera de exponer tiene la desventaja de ocultar la etapa de análisis, de dar la impresión de que el comentarista es un lector muy sagaz, que 
en un primer asalto al texto descubre todo aquello de que nos habla.

Por estas razones, yo voy a seguir el orden del análisis, con la intención de ofrecer los materiales para una lectura más atenta del texto que va a ocupar mi atención hoy y que dice así:

AFECTOS VARIOS DE SU CORAZÓN

FLUCTUANDO EN LAS ONDAS DE LOS CABELLOS DE LISI

En crespa tempestad del oro undoso nada golfos de luz ardiente y pura mi corazón, sediento de hermosura,

4 si el cabello deslazas generoso.

Leandro, en mar de fuego proceloso, su amor ostenta, su vivir apura; Ícaro, en senda de oro mal segura,

8 arde sus alas por morir glorioso.

Con pretensión de fénix, encendidas sus esperanzas, que difuntas lloro,

11 intenta que su muerte engendre vidas.

Avaro y rico y pobre, en el tesoro, el castigo y la hambre imita a Midas,

14 Tántalo en fugitiva fuente de oro.

(Francisco de Quevedo, Obra poética. Edición de José Manuel Blecua. Madrid, Castalia, 1969, I, soneto 449, páginas 644-645).

En el nivel fónico del texto hago, después de una lectura atenta, las cuatro observaciones siguientes. Primera: partiendo de una pura intuición, parece predominar la vocal $E$ (vocal clara, según D. Alonso). Basándose en la estadística, se llega al siguiente resultado: de 384 fonemas que constituyen el poema, 53 corresponden al fonema $e$, lo que representa el 13,8 por ciento. Este porcentaje es ligeramente superior a la media de la aparición del fonema $e$ en la lengua española, que es del 11,75 por ciento según Navarro Tomás (V. Estudios de fonologia española, p. 17). Indudablemente, palabras como tempestad, sediento, pretensión, esperanzas, muerte, engendre, fuente, contribuyen a este efecto y se refuerzan, al mismo tiempo, dentro de este predominio del fonema e. ¿Qué valor simbólico puede tener este hecho? Si seguimos a D. Alonso, la claridad atribuida al fonema $e$ se tendría que poner en relación con el significado claro, o con la connotación de valoración positiva del tema: hermosura de los cabellos rubios. 
La segunda observación se refiere al final del verso 1, que se configura como pasajes en que se impone el sonido: oro undoso. Aunque el valor oscuro que puede tener la vocal $o$-unido, además, al mismo valor que puede tener la vocal $u$ - parece estar en contradicción con el significado y la valoración positiva de ORO, no olvidemos la asociación que puede hacerse entre la vocal o y lo rotundo, lo maravilloso - piénsese en la interjección iOH!-. Además, y con esto hago una incursión en el campo de la sintaxis, encontramos aquí juntas una rima que aparecerá en los tercetos, -ORO, y que asuena con una de las rimas consonantes de los cuartetos, -OSO. La palabra ORO se repite en la posición final del soneto. Es, pues, una palabra que se realza fonéticamente, posicionalmente, y que, semánticamente, ocupa un lugar primordial para significar el cabello. En posición central del verso central del soneto (sílabas 6-7 del verso 7) volvemos a encontrar la palabra ORO.

La tercera observación se refiere a la paronomasia del verso 13: imita a Midas. Parece como si la significación de «imitación» estuviera simbolizada en la repetición de la sílaba MI.

Por último, señalo la aliteración del verso 14 en el sintagma fugitiva fuente. La suavidad de las consonantes fricativas $-f$ en este caso-, aparte del valor onomatopéyico de la $f$-soplar, fluir-, crea un conjunto fónico que puede ser relacionado simbólicamente con el significado de FUGITIVA FUENTE: fuente que mana.

Paso a comentar el nivel sintáctico del texto. Por lo que respecta a la estructura de la frase, señalo, dentro de los procedimientos elípticos, la supresión del sujeto (mi corazón) en los dos tercetos. En el segundo terceto, sin embargo, avaro, rico y pobre pueden interpretarse como sustantivos y entonces están en la misma relación metafórica con corazón que están Leandro, licaro, en el segundo cuarteto. A no ser que Tántalo signifique también mi corazón, siguiendo así el paralelismo del segundo cuarteto. De todas formas, parece que no puede afirmarse que el soneto sea excesivamente concentrado, pues casi todos los elementos están explicitos.

Entre los procedimientos sintácticos que suponen una ampliación de la estructura de la frase señalo el polisindeton del verso 12: avaro y rico y pobre, con el efecto de lentitud que produce, reforzado en este caso con la enumeración que sigue: en el tesoro, el castigo y la hambre.

Por lo que se refiere a la ordenación artificiosa de los elementos de la frase, señalo, en el primer verso, la disposición de sustantivo y adjetivo en forma de quiasmo:

\section{en crespa (1) tempestad (2) del oro (2) undoso (1).}

Entre los tres primeros versos del soneto se da una inversión total, pues estos versos se leen en su orden normal si se empieza por el tercero y se va hacia el primero: mi corazin. sediento de hermosura, I nada golfos de luz ardiente y pura / en crespa tempestad del oro undoso. 
El hipérbaton es, sin duda, el recurso más ampliamente utilizado en este soneto. Este fenómeno puede afectar a la ordenación de los elementos dentro de un verso, o a la ordenación de los elementos de un período que abarca varios versos. Ejemplos de hipérbaton referidos a un solo verso lo constituyen los versos $4,6,10$ y 13 . Referido a varios versos, encontramos ejemplos de hipérbaton en los versos 1-4, 5-6, 7-8, 9-11 y 12-14. Observemos que ni un solo período está libre de hipérbaton. Este hipérbaton tan abundante puede ser compensado por la frecuencia de paralelismos y emparejamientos.

Por lo que se refiere a los paralelismos, hay uno perfecto entre los versos 5,7 y 14, basado en el esquema sustantivo + complemento circunstancial. Más perfecto es el paralelismo entre los versos 5 y 7 , pues este paralelismo se extiende a la ordenación de los elementos que conforman el complemento circunstancial: en + sust. + de + sust. + adj.

Paralelismo sintáctico en la ordenación de cada una de las dos partes del verso, se encuentra en el verso 6: su amor ostenta, su vivir apura. Es el fenómeno que D. Alonso llama bilateralidad, al que se une la epanáfora de SU (repetición al principio de varias frases). Otros casos de paralelismo tendremos ocasión de ver al estudiar la métrica y los emparejamientos.

Voy a reunir en este momento todas las observaciones que se pueden hacer a propósito de la métrica de este poema. Y esto porque pienso que la métrica se sitúa perfectamente dentro de los fenómenos sintácticos, fenómenos de orden sintagmático.

Un solo caso de encabalgamiento hay en el soneto. Se trata del encabalgamiento suave entre los versos 9 y 10: encendidas/sus esperanzas. El efecto de relevancia se produce sobre la palabra esperanzas, por cuanto que encendidas entra coherentemente dentro del campo de fénix -asociado con las cenizas y el fuego-y cobra su sentido metafórico al romperse la coherencia con la aparición de la palabra esperanzas al principio del verso 10.

La acentuación extrarrítmica en primera sílaba del verso pone en relación las siguientes palabras: nada (v. 2) y arde (v. 8), que son empleos transitivos de verbos intransitivos; Ícaro (v. 7) y Tántalo (v. 14), que son nombres propios esdrújulos y metáforas de mi corazón.

La acentuación rítmica en 6." sílaba es la que caracteriza a este soneto, pues todos los versos-excepto el 6,8 y 10-tienen acentuada esta sílaba. En esta posición se acentúan las siguientes palabras: tempestad, luz, sediento, deslazas, fuego, oro, fënix, muerte, pobre, hambre, fugitiva. Estas palabras pueden relacionarse de la siguiente manera: oposición entre tempestad y luz; redundancia de los semas de calor y color en las palabras fuego y oro; relación referencial entre fuego, fénix y muerte.

Dentro todavia del comentario de la acentuación del soneto, señalo que en el verso 5 hay dos partes claramente diferenciadas: la primera, con acentuación yámbica de fuerte ritmo marcado inacentuada-acentuada: Leándro en már de fuégo; la segunda, nada más que con el acento obligatorio en 
décima silaba. En este verso se inicia una estrofa, y semánticamente puede señalarse la oposición entre mar y fuego.

Esquema semejante al comentado en el verso 5, encontramos en el v. 12: una primera parte con ritmo yámbico: aváro y rico y póbre; la segunda, con sólo el acento obligatorio en $10^{\mathrm{a}}$. Además, hay dos sinalefas, unidas al polisíndeton, en las que intervienen las mismas vocales oy. Semánticamente, hay oposición entre rico y pobre, y asociación hasta cierto punto sinonimica entre avaro y rico.

También merece un pequeño comentario la rima del soneto. Cuatro de las palabras que constituyen una de las rimas de los cuartetos (la rima en -oso) asuenan con las palabras que forman una de las rimas de los tercetos (la rima en -oro). La rima en -ura está formada por cuatro palabras que pueden oponerse dos a dos: unas signitican lo positivo (pura, hermosura); y las otras dos, lo negativo (apura, mal segura). La rima en -oro une a las palabras Iloro, tesoro, oro: entre tesoro y oro se da una relación metonímica; y la valoración positiva de estas palabras se opone a la negativa de lloro. La rima en -idas une a las palabras encendidas, vidas, Midas, que tienen connotación positiva.

En primera posición de verso se encuentra el sujeto de todo el sonetomi corazón (v. 3)-y prácticamente todos los personajes míticos que sustituyen metafóricamente a corazón en los desarrollos posteriores -faltan de esta posición fénix (v. 9) y Midas (v. 13)-. Ya he señalado antes la relación, por su acentuación extrarrítmica, entre Icaro y Tántalo.

En los versos 12-13 se establece una correlación que puede representarse de la siguiente forma:

$\begin{array}{cccl}1 & 2 & 3 & \\ \text { avaro } & \text { rico } & \text { pobre } & \text { [mi corazón] } \\ \downarrow & \downarrow & \downarrow & \text { Midas } \\ \text { tesoro } & \text { castigo } & \text { hambre } & \text { Mamber }\end{array}$

Entro seguidamente en el análisis de los emparejamientos en posiciones comparables y en posiciones paralelas. En posiciones comparables -las ocupadas por palabras que desempeñan la misma función dependiendo del mismo núcleo- señalo los siguientes casos: en el v. 2 , ardiente y pura son epitetos que se refieren a luz y que están dispuestos siguiendo un marcado ritmo yámbico. En el v. 6, su amor ostenta, su vivir apura son dos frases que se refieren a Leandro. Ya he señalado la bilateralidad de este verso, la epanáfora de SU, y ahora añado la relación semántica establecida por las connotaciones positivas de amor y vivir, y la oposición entre ostenta y apura. En el v. 12 , avaro y rico y pobre desempeñan la misma función respecto al sobrentendido «mi corazón». Fonéticamente, estas tres palabras constituyen un heptasilabo de ritmo yámbico muy marcado. También desde el punto de vista fónico, cabe señalar la alternancia de vocales claras y oscuras, dándose en el 
centro la diptongación de una vocal oscura (o) y una clara (i). El conjunto está rodeado de vocales claras. En esquema: a a oi $i$ io o $e$. De las relaciones semánticas entre estas palabras hemos hablado al tratar la métrica. En los versos 12-13, en el tesoro, el castigo y la hambre desempeñan la misma función respecto de imita a Midas. Cabe señalar la oposición semántica entre la valoración positiva de tesoro y la negativa de castigo y hambre.

En posiciones paralelas -las ocupadas por palabras que desempeñan la misma función respecto de núcleos diferentes-la abundancia de emparejamientos es perceptible en una primera lectura. Paso a señalar gráficamente en un cuadro el paralelismo entre las construcciones que se encuentran en los versos $1,5,7$ y 14 .

\begin{tabular}{|l|l|l|l|l|l|l|}
\hline & Prep. & Adj. & Sust. & Prep. & Sust. & \multicolumn{1}{|c|}{ Adj. } \\
\hline v. 1 & en & crespa & tempestad & del & oro & undoso \\
\hline v. 5 & en & & mar & de & fuego & proceloso \\
\hline v. 7 & en & & senda & de & oro & mal segura \\
\hline v. 14 & en & fugitiva & fuente & de & oro & \\
\hline
\end{tabular}

Todos los elementos que entran en el paralelismo se encuentran en el primer verso. Por otra parte, en esta serie de emparejamientos se encuentran todas la metáforas referidas a cabello, y que son:

crespa tempestad del oro

proceloso mar de fuego

mal segura senda de oro

fugitiva fuente de oro.

Leyendo verticalmente, se forman los siguientes grupos de palabras: 1. crespa, proceloso, mal segura, fugitiva: adjetivos que indican movimiento inseguro y peligroso; 2. tempestad, mar, senda, fuente: son los núcleos de las metáforas de cabello, y tienen un significado común de movimiento; 3. oro, fuego, oro, oro: sustantivos precedidos de la preposición de con función de adjetivos, y que suponen una iteración tópica sobre el color rubio del cabe1lo. Fuera de esta relación queda el adjetivo undoso, que se relaciona asociativamente con el movimiento del cabello y del mar, la senda y la fuente; y, por otra parte, no tiene la valoración negativa de los otros adjetivos del grupo, y se convierte en el fundamento de todas las relaciones que se establecen entre cabellos y tempestad, mar, senda y fuente.

Como sujetos de distintos verbos se ponen en paralelo las siguientes palabras: mi corazón (v. 3), Leandro (v. 5), Icaro (v. 7). En este paralelismo funcional encuentro explicitados el término metaforizado (mi corazón) y los 
términos metafóricos (Leandro, İcaro). Antes he comentado la relación posicional que se establece entre estos términos en la matería del poema.

Los adjetivos del poema desempeñan función paralela respecto de distintos núcleos. Estos adjetivos son: crespa, undoso, ardiente, pura, sediento, generoso, proceloso, mal segura, glorioso, encendidas, difuntas, avaro, rico, pobre, fugitiva. Pues bien, algunos de estos adjetivos se relacionan fónicamente por rimar entre sí: undoso, generoso, proceloso, glorioso, que son todas las palabras que constituyen la rima en -OSO. Riman también pura y mal segura. Semánticamente, el significado de pasión, tensión y movimiento es común a muchos de estos adjetivos: crespa, undoso, ardiente, sediento, generoso, proceloso, glorioso, encendidas, avaro, fugitiva. Está claro que entre los adjetivos predominan los que indican tensión fisica o espiritual, pasional.

El soneto tiene sus elementos muy relacionados sintácticamente por medio de emparejamientos. De todas formas, hay que observar que no todas las partes del mismo se encuentran igualmente trabadas. En este sentido, es muy clara la diferenciación del primer terceto respecto del resto del soneto. En una primera lectura se aprecia ya este distinto carácter. Carácter que se confirma si comprobamos, como resultado de un análisis sintáctico, que en este terceto hay una abundante subordinación (participio absoluto, oración de relativo y oración completiva, tipos de oraciones que no se encuentran en el resto del soneto). Este terceto, pues, da cierta variedad al ritmo sintáctico del soneto que, de otra forma, produciria, quizá, un paralelismo excesivo, machacón y pesado.

Toca analizar seguidamente el nivel de la significación del texto. Aunque al hilo del análisis de la sintaxis se han deslizado observaciones que tenian que ver con la significación. Esto ocurría, por ejemplo, al estudiar los emparejamientos.

Empiezo analizando las metáforas, y sigo el mismo orden de su aparición en el texto. El adjetivo crespa (v. 1) no es una metáfora, pues es un término que puede aplicarse lo mismo a pelo rizado y hueco que a cosas de aspecto arrugado -en este caso, tempestad-. Bien es verdad que el adjetivo crespo, por poder aplicarse a cabello y a tempestad, refuerza la metáfora de tempestad para significar cabello en movimiento, que analizo seguidamente. Tempestad significa metafóricamente melena en movimiento. La forma arrugada de la superficie del mar cuando hay tormenta, y esta misma forma del pelo crespo es el fundamento de tal metáfora. El adjetivo crespo, según he dicho antes, selecciona los semas comunes a melena y a tempestad. También refuerza esta significación el adjetivo undoso, común a tempestad y a cabello que se mueve.

El verso 2 se inicia con la metáfora de nada para el significado de quedar suspendido, flotando -ien el sentido de quedar embelesado?-. La falta de apoyo sólido es lo común al significado de nadar y quedar suspendido o embelesado. El empleo de nadar tiene su justificación en las metáforas de tipo acuático referidas a cabello y que se inician con tempestad. 
En la frase "golfos de luz ardiente y pura» encuentro la metáfora de golfos con el significado de rizos, que se fundamenta en la común forma redondeada, y la de "de luz ardiente y pura» con el signiticado de «luminosos, relumbrantes»'.

El empleo metafórico de "sediento" por "deseoso" hoy se siente como lexicalizado. Bien es verdad que la utilización de sediento, que en sentido real significa "deseoso de agua", entra coherentemente dentro de la presentación del cabello como mar. En este caso se trataría de un deseo de beber el agua del cabello.

El segundo cuarteto se inicia con la primera de una serie de metáforas referidas a "mi corazón» y que tienen que ver con personajes mitológicos. El hecho de que «Leandro» pueda sustituir a «mi corazón» se fundamenta en que tanto el uno como el otro tienen que ver con el mar: Leandro, en sentido real, según la leyenda; mi corazón, en sentido metafórico, en cuanto que el cabello es tempestad y golfos. Los dos, además, están enamorados.

Continúa el verso 5 con la metáfora del «mar de fuego proceloso» para referirse al cabello en movimiento y de color rubio. La idea de movimiento viene dada no sólo por la sustitución de cabello por mar, sino también por la utilización de la metáfora «de fuego» para referirse al color del pelo. Pues el fuego indica color dorado, movimiento y fuente de calor (pasión). El pelo también es fuente de pasión.

La segunda referencia a un personaje mitológico con el que comparar al corazón aparece en el verso 7. El fundamento para que "Ícaro" sustituya a mi corazón está en que la acción de elevarse con peligro de quemarse se aplica a İcaro en sentido real y al corazón que corre peligro de arder por la pasión despertada por el cabello.

En «senda de oro» encontramos otra metáfora para referirse al cabello rubio. Senda de oro, referido a Ícaro significa el camino del sol, lugar de su desgracia. Igualmente, para el corazón enamorado los cabellos de oro son lugar de perdición ${ }^{2}$.

En el primer terceto, encontramos la metáfora de encendidas con el significado de enardecidas, incitadas, aunque es cierto que este sentido de "encender" está prácticamente lexicalizado. Ahora bien, encendidas se puede

1 Rizo se aplica al pelo, pero también se aplica rizar a uformar ondas en el agua el viento, la caida de algo, etc.». En este sentido es asociable con crespo, aplicable al mary al cabello. Hay un refrán en el que aparecen las palabras nadar y golfos, aunque con sentido diferente del que tienen en el texto. Dice el refrán: Este mundo es golfo redondo, quien no sabe nadarvase a to hondo. (Diccionario de Autoridades, s.v. nadar).

2 Arde sus alas por morirglorioso:se lee en sentido real referido a la leyenda de Ícaro. Referido a corazón, indica la primacia del ansia de gloria, de elevación sobre el deseo de seguridad. Respecto al uso transitivo de este verbo dice el Diccionario de Autoridades: «Suele usarse como verbo activo, aunque pocas veces, $y$ vale lo mismo que abrasar, o quemarn. Y da el siguiente ejemplo de Quevedo, Zahurdas: «De qué te quexas si ninguno te molesta? Si el fuego no te arde, ni el hielo te cerca?» s.v. arder. 
leer en sentido real refiriéndose al ave fénix, y entonces esperanzas significa "cuerpo" (del ave fénix que arde). A partir de aquí, difuntas se puede leer en sentido real referido al cuerpo del ave fénix, y en sentido metafórico referido a esperanzas; y entonces da lugar a una metáfora en que difuntas significa "sin existencia real", fundamentándose en la falta de actividad y futuro. Así se opone a encendidas ${ }^{3}$.

En el verso 13 encuentro una metáfora muy interesante. Se trata del uso del verbo imitar para significar "ser como". El fundamento de este uso metafórico es la expresión de la semejanza. Y es interesante esta metáfora porque es la metáfora de la expresión lingüística de la relación metafórica. Compárese, si no, esta construcción con las semejantes: Leandro, en mar...; Ícaro, en senda...; Tántalo en fugitiva fuente... Si suprimimos «imita a» tendríamos una expresión paralela completamente: Midas, en el tesoro, el castigo y la hambre.

En el tesoro, el castigo y la hambre se leen en sentido real referidos a $\mathrm{Mi}$ das, pero, referidos a corazón, tienen un significado metafórico que podemos explicar así: tesoro significa cabello, fundamentándose en el color dorado; el castigo, en cuanto que produce dolor, significa también cabello; el hambre significa carencia, por cuanto que en ambos términos hay una insatisfacción del deseo. Los adjetivos con que se inicia este terceto tienen, entonces, un sentido real referidos a Midas, pero cobran un sentido metafórico referidos a corazón. Este sentido metafórico puede explicarse de la siguiente manera: avaro significa nunca satisfecho, basándose en la existencia de un deseo desordenado; rico quiere decir que se le ofrece el cabello de oro, siendo el fundamento la abundancia de oro-cabello; pobre quiere decir que no tiene el cabello, funcionando como fundamento la falta de posesión ${ }^{4}$.

3 Intenta que su muerte engendre vidas: referido al ave fénix, puede leerse en sentido real. Referido a corazón, significa su intento de que surjan unas esperanzas ya muertas, y entonces vidas=esperanzas. Otro ejemplo de alegoría referida al ave fénix se encuentra en el soneto 450 , versos 1-4, de Quevedo:
Hago verdad la fénix en la ardiente
llama, en que renaciendo me renuevo,
y la virilidad del fuego pruebo,
y que es padre, y que tiene descendiente.

4 Estamos aqui en un terreno en el que es muy dificil, si no imposible, explicar lingüisticamente la gran riqueza significativa de las relaciones que se establecen recurriendo exclusivamente a la metáfora - a lo meramente lingüistico-, dejando aparte la realidad exterior, que entraria en las explicaciones de tipo alegórico. Ejemplo de esta explicación de tipo alegórico es la que da Alexander A. Parker: «El sentido, según creo, es: «Por ser avaro y rico y pobre, imita a Midas en el tesoro, en el castigo y en el hambre». Es decir: Por ser avaro de hermosura mi corazón imita a Midas en querer tener el tesoro; por ser rico en tener presente el oro imita a Midas en el castigo (porque este no podia aprovecharse de su tesoro); por verlo y no poseerlo, mi corazón es pobre a la vez que rico, y, por consiguiente, imita a Midas en el hambre que padecia (porque todo lo que trataba de comer se volvía oro al tocarlo sus labios)". V. "La agudeza" en algunos sonetos de Quevedo", pág. 351, n.I. 
El último verso recoge dos metáforas: una referida a corazón y otra referida al cabello. En Tántalo se encuentra el último personaje mitológico con el que es comparado el corazón, fundándose en que tanto uno como otro son seres castigados. Fugitiva fuente de oro significa la melena rubia en movimiento. Tanto el movimiento como el efecto visual del pelo agitado por el viento, que a veces se ha comparado con los hilos del agua, sirven de fundamento para tal comparación. En la alegoría, tanto la fuente para Tántalo, como el cabello para mi corazón son causa de suplicio. El término "fugitiva fuente», referido a la leyenda de Tántalo, se lee en sentido real, pero la aparición del sintagma «de oro" nos conduce a la lectura metafórica dentro del significado de cabello.

Los cambios de significado basados en el desplazamiento dentro de la contigüidad son mucho menos abundantes que los cambios basados en la metáfora. Dos cambios de tipo metonímico están presentes en todo el soneto. El primero es la utilización de oro para significar "cabello de color de oro", tomando el contenido por el continente. Que se refiere a cabello sin lugar a dudas viene fundamentado en la aparición de crespo y undoso, términos aplicables en sentido real al cabello. Esto sin hablar de la práctica lexicalización de oro para referirse a cabello rubio en poesía. El segundo cambio es el de «mi corazón» por toda mi persona, «yo». Se trata de utilizar una parte por el todo. Mi corazón puede interpretarse también, dentro de una perspectiva muy realista, como metáfora por amor, siendo el fundamento la localización tópica del sentimiento en esta parte del cuerpo.

Como se ha podido ver, muchos de los elementos lingüísticos figurados tienen una lectura en sentido real referidos al elemento con el que se compara el corazón. Pero siempre hay un elemento que rompe esta coherencia referencial, que se refiere al otro plano y que desencadena la doble lectura propia de la alegoría. Así, de oro, de fuego, son elementos que se refieren a cabello rubio y que arrastran los demás significados hacia una significación coherente dentro de esta referencia al cabello.

Una vez estudiados los cambios de sentido, voy a intentar analizar las iteraciones de sentidos producidas en el texto. Asociándolas por su significado, pueden agruparse las palabras del texto en los siguientes grupos: primero, el de las palabras que se refieren a lo liquido y en el que entraría tempestad, nada, golfos, sediento, mar, fugitiva fuente; segundo, el de las que significan movimiento - tanto físico como tensión espiritual-y en él estarían las palabras crespa, undoso, nada, deslazas, sediento, ostenta, apura, senda, mal segura, arde, pretensión, encendidas, esperanzas, intenta, engendre, avaro, hambre, imitar, fugitiva fuente. Un tercer grupo sería el de las palabras referidas al color-dorado y suntuoso-: oro, luz ardiente y pura, hermosura, generoso, de fuego, de oro, glorioso, rico, tesoro, fuente de oro. Por último, podría formarse un grupo con los nombres de personajes mitológicos: Leandro, Ícaro, Fénix, Midas, Tántalo, personajes todos simbólicos de un padecer y de una tensión.

Resumen de estos campos asociativos en que, subjetivamente por su- 
puesto, agrupo la mayoría de los términos del soneto puede ser el verso final:

\section{Tántalo en fugitiva fuente de oro}

donde se encuentra: lo liquido (fuente), el movimiento fisico (fugitiva fuente) y tensión espiritual (Tántalo), el color (de oro), y la mitología (Tántalo). El campo mitológico, constituido por personajes asociados al padecer y a la tensión, puede integrar un subgrupo dentro del grupo formado por los significados de movimiento físico y tensión espiritual. El último verso, constituye, pues, un broche de oro para el tema del soneto. Por supuesto que el título mismo ya recoge los diversos aspectos del tema: lo líquido (ondas), movimiento (afectos varios, fluctuando), lo suntuoso (cabellos de Lisi).

$\mathrm{Al}$ analizar el texto partiendo de la realidad configurada en el mismo, voy a fijarme especialmente en tres grupos de cuestiones: figuras que tienen que ver con una configuración de la realidad poetizada; descripción de la experiencia objeto del soneto; inserción del tema y su tratamiento dentro de una contexto histórico literario.

Al hablar de los emparejamientos hubo ocasión de ver la gran cantidad de relaciones sintácticas que se establecian dentro del poema. Las palabras emparejadas frecuentemente se relacionaban por una oposición de sus significados. Dentro de esta tendencia a la oposición se sitúan también figuras como la antitesis - contraposición de dos pensamientos de amplitud sintáctica variable-, o como la paradoja - unión de dos ideas aparentemente irreconciliables-. Ejemplos de antitesis, en el soneto, son: su amor ostenta, su vivir apura (v. 6); encendidas sus esperanzas que difuntas lloro (vv. 9-10). Como ideas paradójicas pueden citarse: mar de fuego (v. 5); intenta que su muerte engendre vidas (v. 11); rico y pobre (v. 12).

Más importancia tiene, sin duda, en relación con la realidad poetizada, el uso continuo de alegorias mitológicas: Leandro, İcaro, Ave Fénix, Midas, Tántalo. Al estudiar las metáforas se observó cómo hechos que se leian en su sentido real referidos a los personajes mitológicos y su realidad legendaria, eran insertados metafóricamente dentro de la realidad espiritual, dentro de los sentimientos de su corazón.

La realidad representada en este soneto ha sido descrita por los comentaristas del mismo. Alexander A. Parker lo hace en los siguientes términos:

"La experiencia en que se basa el soneto no puede ser más sencilla: el poeta mira a la mujer amada deslazar el cabello. Este se transforma en seguida mediante cuatro concepros: 1) por estar suelto es un golfo o mar, $y$ por ser $r$ zado es un mar de ondas agitadas que amenazan muerte al nadador; 2) por ser dorado es el oro material, o sea un tesoro codiciable, y también 3) la luz del sol y el espacio que atraviesan su rayos;4) siendo los rayos del sol, es fuego que quema y mata. ${ }^{5}$

5 Véase. art. cit. pág. 351. 
También José María Pozuelo Yvancos resume el tema de este soneto en los siguientes términos:

"La nimiedad del motivo que origina el poema contrasta una vez más con la potente afluencia poética del lenguaje de Quevedo. Este es un rasgo típico de su lírica: a partir de experiencias minúsculas construir, desautomatizando las relaciones habituales y el sistema expresivo, poemas de gran trascendencia simbólica. Lisi se deslaza el cabello (v.4) y a partir de ahí se llega a la manifestación más radical de la incomunicabilidad del sentimiento y la frustración de la comunicación amorosa.» ${ }^{6}$

Nada debo añadir yo a las palabras de estos dos estudiosos de la obra de Quevedo, respecto a la realidad representada, poetizada en el soneto.

Todo texto literario hereda y modifica un legado anterior. En este sentido, el texto se refiere también a una realidad ya conformada anteriormente por otros textos literarios. Por eso puede hablarse del texto como «mosaico de citas". El texto afirma y niega el conjunto de textos coetáneos y anteriores. Afirma en cuanto que toma algo de ellos -desde un rasgo estilístico hasta un tema-, y niega en cuanto que, frente a los demás textos, tiene siempre algo de distinto y original. El estudio de estas relaciones introduce de lleno en cuestiones de historia literaria. Yo me voy a limitar a la ilustración de estas relaciones del soneto que estoy comentando con otros textos del mismo Quevedo. Tomo como ejemplo algunas correspondencias estilísticas sorprendentes que demuestran hasta qué punto el poema afirma otros poemas del mismo Quevedo.

En el soneto 349, titulado «A Fili, que suelto el cabello, lloraba ausencias de su pastor", se lee:

Ondea el oro en hebras proceloso; corre el humor en perlas hilo a hilo; juntó la pena al Tajo con el Nilo, este creciente, cuanto aquel precioso. Tal el cabello, tal el rostro hermoso asiste en Fili al doloroso estilo, cuando por las ausencias de Batilo, uno derrama rico, otro lloroso.

En el verso 12 de este mismo soneto se encuentra una aliteración igual a la que vimos en «imita a Midas». Alli se puede leer:

Dijo: «Si imitas mi dolor constante».

6 Véase, El lenguaje poético en la lirica amorosa de Quevedo, págs. 148-149. 
(Esto dice Fili a una tórtola amante que oyó gemir «con músico lamento y mustia y ronca voz».)

En el poema 385 , titulado «Celebra el cabello de una dama, que habiéndosele mandado cortar en una enfermedad, ella no quiso", se lee en los versos 53-54:

Invidia sea del sol, desprecio al sol, prisión a l'alma, y al amor tesoro.

Estos versos se refieren al cabello. Del poema 409, titulado «Alma en prisión de oro", leo los versos 1-4:

Si alguna vez en lazos de oro bellos la red, Flori, encarcela tus cabellos, digo yo, cuando miro igual tesoro, que está la red en red y el oro en oro.

Transcribo, finalmente, otro soneto a Lisi, el 501, titulado «A Lisi que en su cabello rubio tenía sembrados claveles carmesíes, y por el cuello».

Rizas en ondas ricas del rey Midas, Lisi, el tacto precioso, cuanto avaro; arden claveles en su cerco claro, flagrante sangre, espléndidas heridas.

Minas ardientes, al jardin unidas, son milagro de amor, portento raro, cuando Hibla matiza el mármol paro $y$ en su dureza flores ve encendidas.

Esos que en tu cabeza generosa son cruenta hermosura y son agravio a la melena rica y victoriosa, dan al claustro de perlas, en tu labio, elocuente rubi, púrpura hermosa, ya sonoro clavel, ya coral sabio.

Como puede apreciarse, unas mismas palabras -o palabras del mismo campo semántico- aparecen en Quevedo para referirse a una misma realidad poética. La poesía de Quevedo es una poesía pensada: piensa en cosas similares -tesoro, Midas...-cuando se refiere a temas similares: cabello.

Que estos pensamientos se sitúan en un contexto poético exterior a Quevedo, nos lo demuestra el hecho de que en Garcilaso o en Góngora 
puede encontrarse igualmente la referencia al cabello como oro. Recuérdese el famoso soneto de Garcilaso "En tanto que la rosa y d'açucena" cuyos versos 5 y 6 dicen:

$y$ en tanto que el cabello, que en la vena del oro escogio, con vuelo presto.

O recuérdense los dos primeros versos del famoso soneto de Góngora:

Mientras por competir con tu cabello oro bruñido el sol relumbra en vano.

Esta asociación del oro y el cabello rubio viene del petrarquismo. Sirva esta apreciación de enlace para ver cómo contextualiza José María Pozuelo este poema, y señala lo que tiene de deuda con el petrarquismo, con la concepción del amor cortesano, $y$ lo que tiene de original -cómo desautomatiza la temática y estilo heredados:

\footnotetext{
"La base del "concepto", y el núcleo semántico generador del poema, es el contraste entre la posesión distante de atributos fisicos (el cabello de Lisi se admira) y la incomunicación definitiva a que el amor cortesano (aqui con una indiscutible base neoplatónica) somete al amante, cuyo corazón es puesto en analogía con los mitos del castigo por ambición."
}

La manifestación de la incomunicabilidad se apoya «sobre un intelectualismo y densidad conceptual muy notable», en los que es importante el relieve de los códigos culturales-mitológicos y la orquestación conceptual sobre términos muy semantizados por el petrarquismo. De raíz cortesana es el sentimiento del amor. Lo típico de Quevedo está en la «relación entre campos semánticos físicos y símbolos espirituales», o la referencia al plano espiritual mediante necesidades de urgencia fisica. Quevedo es original también en la exageración de la metáfora heredada: oro $=$ cabello $=$ tempestad. Igualmente original es en la superposición de sentidos nuevos a los usuales en el lenguaje poético de la época. Así, apura puede entenderse como purificar?

En su valoración de este soneto, Alexander A. Parker habla de «ejemplo perfecto, logrado con sumo arte, de la tensión barroca», de que este "maravilloso soneto, hondamente emotivo como pocos, es ante todo poesía intelectual». Y José María Pozuelo valora en los siguientes términos:

7 Véase, op. cit., págs. 149-153. 
"Todo en este soneto es heredado y nada en él lo sentimos como superfluo; toda esa herencia está desautomatizada, incorporada a una expresión poética nueva y original. ${ }^{8}$

Solamente quiero añadir una ilustración explícita de la importancia que para Quevedo tiene el movimiento en la concepción de la belleza. Dice Quevedo en el soneto 321, versos 12-14:

No puede en la quietud difunta hallarse hermosura, que es fuego en el moverse, y no puede viviendo sosegarse.

Por eso, si habla del cabello, la belleza no puede estar más que en la melena que se mueve.

${ }^{8}$ Véase, Alexander A. Parker, art. cit. pág. 353; José María Pozuelo, op. cit., pág. 158. Véase también el análisis que hace del tema Midas en la poesía amorosa deQuevedo, $\mathbf{M}$ Blanco Morel. Se centra en el análisis del soneto 501 , que yo transcribo más adelante, pero se refiere, en páginas 97-98, al último terceto del soneto que yo estoy comentando. V. «Métaphore et paradoxe dans deux sonnets de Quevedo", en Bulletin Hispanique. LXXXV, 1983, págs. 83-103. 


\section{BIBLIOGRAFIA}

Blanco Morel. Mercedes: "Métaphore et paradoxe dans deux sonnets de Quevedo", en Bulletin Hispanique, LXXXV, 1983, páginas 83-103.

Dominguez CAPARRós. José: Introducción al comentario de textos. Madrid, MEC, 1982, 2." edic.

FRENZEL, Elisabeth: Diccionario de argumentos de la literatura universal. Madrid, Gredos, 1976.

Molno, Mauricio: Semántica y poética. (Góngora y Quevedo). Barcelona, Critica, 1977, páginas $168-216$.

NavarRo Tomas, Tomás: Estudios de fonologia española. New York, Las Américas Publishing Company, 1966.

Ovidio: Metamorfosis. Barcelona, Alma Mater, 1964; Madrid, C.S.I.C., 1983, 3 vols.

PARKer. Alexander A.: "La "agudeza" en algunos sonetos de Quevedo". En Estudios dedicados a Menéndez Pidal. Madrid, C.S.I.C., 1952, III, páginas 345-360.

Pozuelo Yvancos, José María: El lenguaje poético de la lirica amorosa de Quevedo. Murcia, Universidad, 1979, páginas 148-159.

Quevedo. Francisco de: Obra poética. Edición de José Manuel Blecua. Madrid, Castalia, 1969, Tomo I.

Ruiz de Elvira. Antonio: Mitologia clásica. Madrid, Gredos, 1975.

Real Academia Española: Diccionario de Autoridades. Edición Facsimil. Madrid, Gredos, 1976, 3. ${ }^{\mathrm{a}}$ reimpresión.

TERRY, A.: "Quevedo and the metaphysical conceit", en Bulletin of Hispanic Studies, XXXV, 1958, páginas 211-222. 